\title{
Analyzing time-varying effects of potential output growth shocks ${ }^{2}$
}

\author{
Ümit Özlale ${ }^{\mathrm{a}, *}$, Levent Özbek ${ }^{\mathrm{b}}$ \\ ${ }^{a}$ Bilkent University, Department of Economics, Bilkent 06800, Ankara, Turkey \\ ${ }^{\mathrm{b}}$ Department of Statistics, University of Ankara, Tandoğan, Ankara 06100, Turkey
}

Received 25 August 2006; received in revised form 6 April 2007; accepted 2 May 2007

Available online 10 May 2007

\begin{abstract}
Employing a state space model, we find for the United States economy that potential output growth rate can deviate from its steady state level for substantially long periods.

(C) 2007 Elsevier B.V. All rights reserved.

Keywords: Potential output; Extended Kalman filter

JEL classification: $\mathrm{C} 32 ; \mathrm{C} 63$
\end{abstract}

\section{Introduction}

Measuring potential output has become the main focus of several studies, especially after the central banks have begun to utilize all available information in the economy to understand price dynamics. However, since potential output series cannot be directly observed and remains to be estimated, finding a suitable detrending method and an appropriate estimation technique emerges as an important issue. In this context, "unobserved components" models, which assume a dynamic structure for the 'unobserved' trend and the cycle components, have been widely employed. Harvey (1985), Watson (1986) and Clark (1987) are some of the earlier studies, which utilized these models. ${ }^{1}$ One key difference in these studies is the

\footnotetext{
The authors would like to thank Athanasio Orphanides and Thomas Laubach for their useful suggestions.

* Corresponding author. Tel.: +90 3122901955.

E-mail address: ozlale@bilkent.edu.tr (Ü. Özlale).

${ }^{1}$ Kuttner (1994) and Gerlach and Smets (1999) also applied these models to the United States economy and European Monetary Union area, respectively.
}

0165-1765/\$ - see front matter (C) 2007 Elsevier B.V. All rights reserved.

doi:10.1016/j.econlet.2007.05.006 
assumption about the potential output growth rate. While some of the papers, including Watson (1986), assume a constant steady state growth rate for the potential output, others, including Harvey (1985) and Clark (1987) allow potential output to deviate from its steady state level for substantially long periods.

This study deals with the above mentioned issue within a state space framework, where actual output is decomposed into its trend and cyclical components. First, we remove the assumption that the parameters of the model stay constant over time. Such a time-varying parameter approach makes the state space model a nonlinear one, where the extended Kalman filter (EKF henceforth) is executed. While we can capture the time variation by using EKF, such a methodology does not allow testing for the statistical significance of the parameters. Thus, for robustness purposes and to allow for formal hypothesis testing, we handle the same problem also in a constant parameter state-space model, where the standard linear Kalman filter is employed. As a result, we find that potential output growth rate can deviate from its steady state level for substantially long periods, consistent with the views of Orphanides and Van Norden (2002). However, we cannot claim that potential output growth rate follows a pure random walk.

\section{The model}

We start by decomposing the actual output into its trend and cycle, such as:

$$
Y_{t}=T_{t}+C_{t}
$$

While the trend component $T_{t}$ can be viewed as the potential output series, the cycle component $C_{t}$ shows the deviation of the actual output from this trend.

To allow for greater business cycle persistence, the cycle term is assumed to follow a second-order autoregressive process with a disturbance term, which has zero mean and constant variance. ${ }^{2}$ Finally, the parameters are allowed to vary over time with random walk:

$$
\begin{aligned}
& C_{t}=\gamma_{1, t} C_{t-1}+\gamma_{2, t} C_{t-2}+\varepsilon_{t} \\
& \gamma_{1, t}=\gamma_{1, t-1}+\zeta_{\gamma 1, t} \\
& \gamma_{2, t}=\gamma_{2, t-1}+\zeta_{\gamma 2, t}
\end{aligned}
$$

It is assumed that $\varepsilon_{t}, \zeta_{\gamma 1, t}$ and $\zeta_{\gamma 2, t}$ are i.i.d. with zero means and constant variances.

About the trend component, there are two basic specifications that are widely used in the literature. Starting with Watson (1986), some studies assume a constant growth rate for the potential output such as:

$$
T_{t}=\mu+T_{t-1}+\eta_{t}
$$

\footnotetext{
${ }^{2}$ All of the papers cited above, including Watson (1986), Harvey (1985), Clark (1987), and Kuttner (1994) assume such a time series specification about the cyclical term.
} 
On the other hand, starting with Harvey (1985) and Clark (1987), some papers specify the trend component as a local linear trend model, which can be written as:

$$
\begin{aligned}
& T_{t}=\mu_{t}+T_{t-1}+\eta_{t} \\
& \mu_{t}=\mu_{t-1}+v_{t}
\end{aligned}
$$

Since the main purpose of this study is to analyze the empirical support of these specifications, we specify the trend component as follows:

$$
\begin{aligned}
& T_{t}=\mu_{t}+T_{t-1}+\eta_{t} \\
& \mu_{t}=\left(1-\rho_{t}\right) \mu_{0}+\rho_{t} \mu_{t-1}+\zeta_{a, t}
\end{aligned}
$$

where the disturbances $\eta_{t}$ and $\zeta_{a, t}$ are again assumed to be i.i.d. with zero mean and constant variances. Eq. (6) defines the trend growth as a first-order autoregressive process with long-run average growth rate of $\mu_{0}$ and an autoregressive coefficient $1>\rho_{t}>0$, representing the persistence in the trend growth. Then, the magnitude of $\rho_{t}$ shows the persistence of the deviations from the long run growth rate, $\mu_{0}$. An estimated $\rho_{t}$ close to one means the potential output growth rate can deviate from the steady state for substantially long periods and vice versa.

\subsection{State space representation}

The simple model introduced above can easily be represented in a state space form, where the state equation and the observation equation are displayed as:

$$
\left[\begin{array}{c}
T_{t} \\
C_{t} \\
C_{t-1} \\
\mu_{t}
\end{array}\right]=\left[\begin{array}{cccc}
1 & 0 & 0 & \rho_{t} \\
0 & \gamma_{1, t} & \gamma_{2, t} & 0 \\
0 & 1 & 0 & 0 \\
0 & 0 & 0 & \rho_{t}
\end{array}\right]\left[\begin{array}{c}
T_{t-1} \\
C_{t-1} \\
C_{t-2} \\
\mu_{t-1}
\end{array}\right]+\left[\begin{array}{c}
1-\rho_{t} \\
0 \\
0 \\
1-\rho_{t}
\end{array}\right] \mu_{0}+\left[\begin{array}{lll}
0 & 1 & 1 \\
1 & 0 & 0 \\
0 & 0 & 0 \\
0 & 0 & 1
\end{array}\right]\left[\begin{array}{c}
\varepsilon_{t} \\
\eta_{t} \\
\zeta_{a, t}
\end{array}\right]
$$

and

$$
Y_{t}=\left[\begin{array}{llll}
1 & 1 & 0 & 0
\end{array}\right]\left[\begin{array}{c}
T_{t} \\
C_{t} \\
C_{t-1} \\
\mu_{t}
\end{array}\right]
$$

The above representation indicates that unobserved state vector $S_{t}=\left[T_{t}, C_{t}, C_{t-1}, \mu_{t}\right]$ and the time varying parameter vector $\theta_{t}=\left[\rho_{t}, \gamma_{1, t}, \gamma_{2, t}\right]$ are to be estimated simultaneously. Moreover, these two sets 


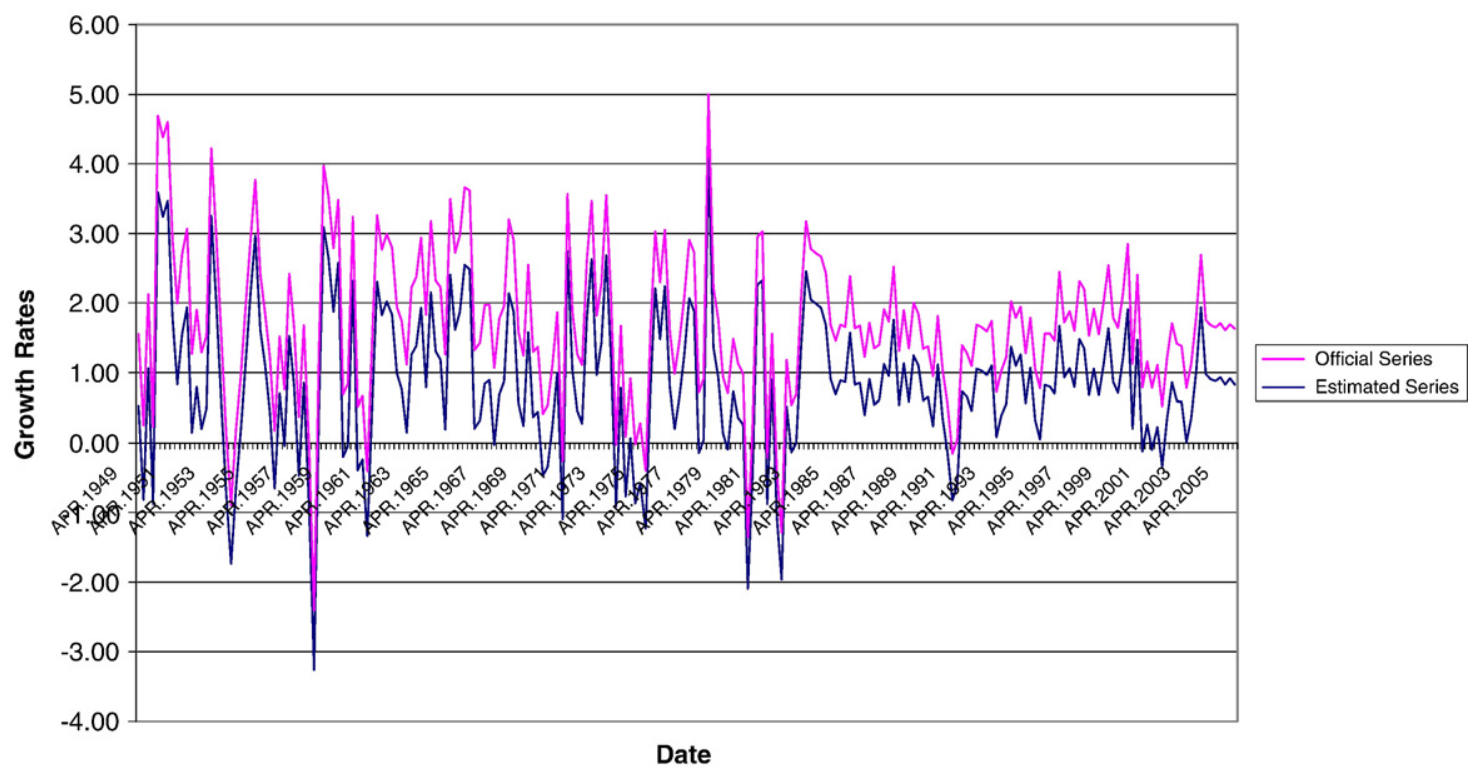

Fig. 1. Comparison of potential output growth rates.

of variables appear in multiplicative form in the state equation. Thus, the problem of nonlinearity emerges, which classifies the model as a nonlinear state space model, and necessitates the use of the extended Kalman filter. As a result, employing the extended Kalman filter, which is specified in Chui and Chen (1991) in detail, the actual output is decomposed into its trend and cycle components while, at the same time, the parameters in the model are estimated in a time-varying fashion.

\section{Estimation results}

\subsection{Sample period and initial values}

For the actual output, we used the U.S. quarterly real GDP from 1949:Q1 to 2005:Q4. The data is seasonally adjusted. We assumed that at the beginning of the sample, the economy was at its potential level and the growth rate for the potential output was equal to its sample average. However, the results did not change even if we relaxed this assumption and tried alternative initial values for the trend and the cycle.

The starting values for $\gamma_{1, t}$ and $\gamma_{2, t}$ are both taken as 0.4 and the persistence parameter $\rho_{t}$ is assumed to have an initial value of 0.5 . As long as the sum for the starting values of $\gamma_{1, t}$ and $\gamma_{2, t}$ did not exceed unity, the results remained robust even when we assumed different initial values for the parameters. In addition, the long run average growth rate $\mu_{0}$ is set equal to the average growth rate of Congressional Budget Office's (CBO) official potential output series. ${ }^{3}$

\footnotetext{
3 Throughout the estimation process, $\mu_{0}$ is constrained to be equal to CBO's official estimate.
} 


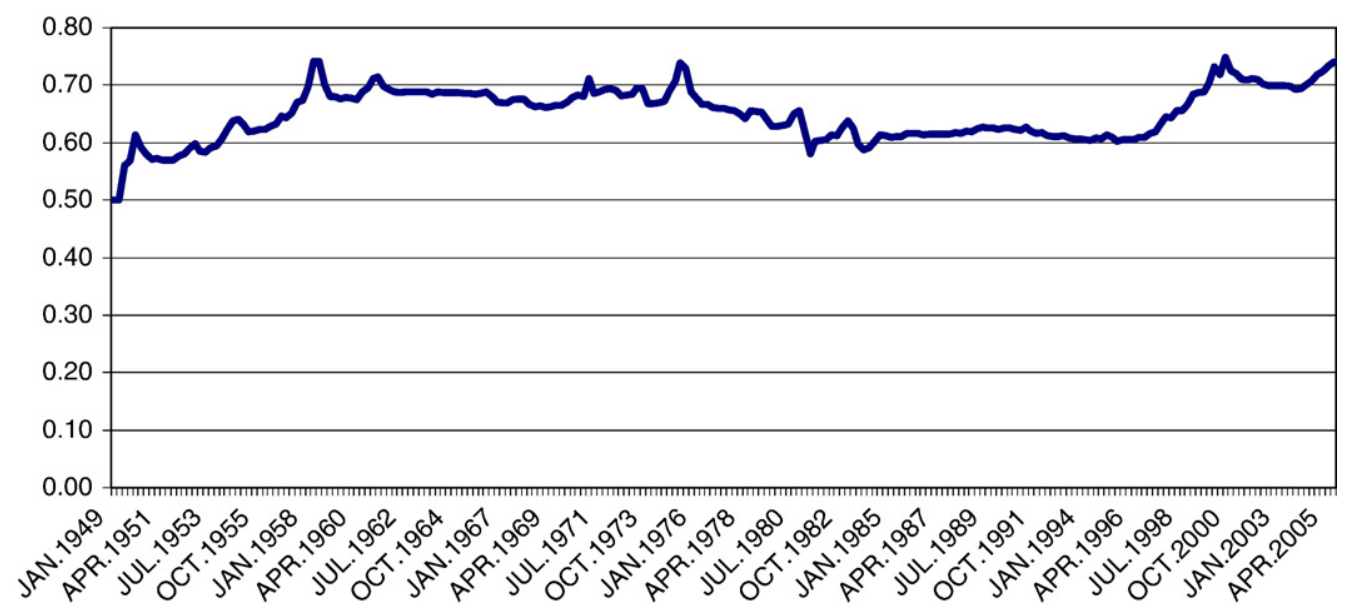

Fig. 2. Estimated persistence.

\subsection{Empirical fit of the model}

The EKF procedure assumes that the disturbance term is normally distributed. After excluding a few observations from the beginning of the sample, where large fluctuations exist due to insufficient number of observations, Jarque-Bera statistics show that the estimated residuals are normally distributed. We also tested whether the estimated cycle term is characterized best by an AR(2) model. We found that the lowest AIC and BIC values are obtained in such a specification. Next, we performed Augmented Dickey Fuller test to analyze whether the time varying parameters follow random walk and found that we cannot reject the null hypothesis of a unit root in the estimated time varying parameters. Finally, we compared our estimated potential output series with CBO's official estimates. As Fig. 1 shows, the growth rates of these two series are very similar. As a result, we can conveniently claim that the underlying specifications about our model are supported by the empirical results.

\subsection{Estimated persistence parameter and the drift}

Fig. 2 displays the time-varying parameter $\rho$, which shows the persistence of deviations from the long run growth rate $\mu_{0}{ }^{4}$ It can be seen that potential output growth is fairly persistent, especially between 1960-1975 and 1999-2005. For example, the average value for the sample period 1999-2005 is 0.71, implying that, in the absence of shocks, output growth would converge within $1 \%$ of the steady-state rate in just about 3.5 years. Moreover, the end-sample estimate for the parameter is 0.74 , implying that shocks continue to have even more permanent effects on the potential output. In fact, the estimated drift parameter in Fig. 3, which has larger swings at the end of the sample, confirms these findings. Consequently, the results that are obtained by using EKF suggest that shocks to potential output growth rate have persistent effects.

\footnotetext{
${ }^{4}$ All of the estimates that are presented in the paper are two-sided estimates.
} 


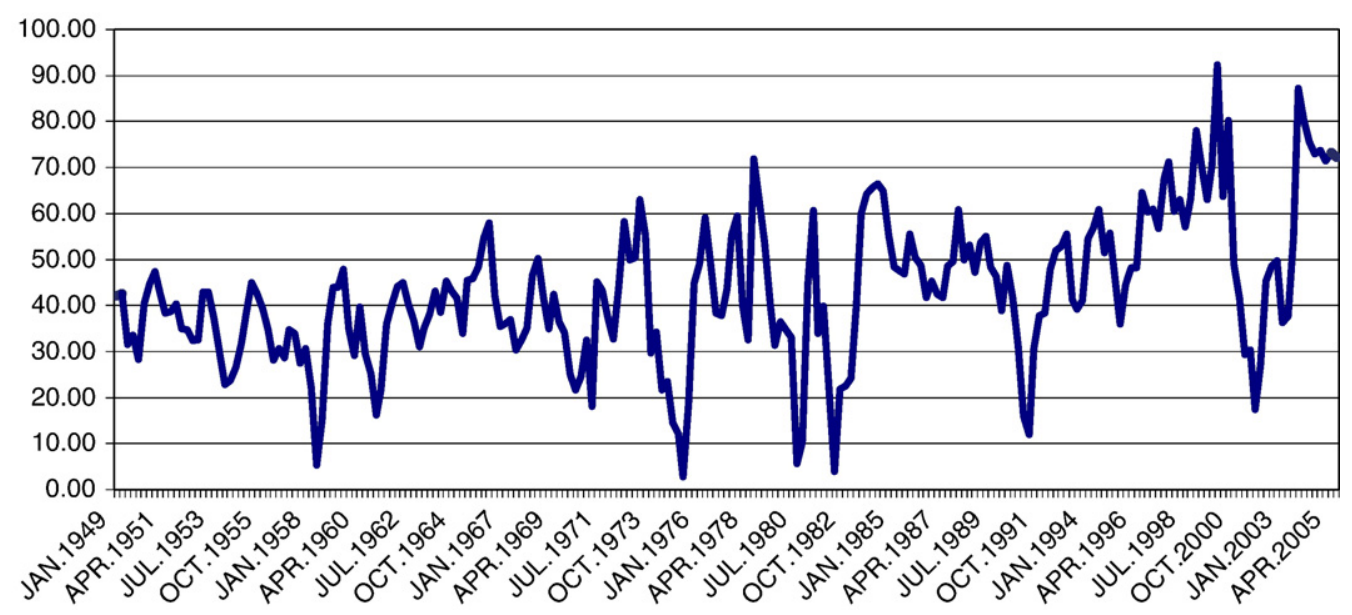

Fig. 3. Estimated drift.

However, as mentioned in the first section, the EKF procedure does not allow testing the statistical significance of the parameters. Thus, in the following part, we employ a constant parameter state space model and perform hypothesis tests.

\subsection{Constant parameter state space model}

When we employ the standard linear Kalman filter for the constant parameter model, the end-sample estimates for $\rho, \gamma_{1}, \gamma_{2}$ are found to be $0.73,0.91$ and 0.09 , with the standard errors $0.06,0.21$ and 0.21 , respectively. Thus, we overwhelmingly reject the null hypothesis that $\rho$ is equal to zero, which implies that a constant steady state growth rate about the potential output is misleading. However, we also reject the null hypothesis that $\rho$ is equal to unity, where the estimated $t$-statistic is slightly above the critical value. As a result, we conclude that, although shocks to potential output growth rate have fairly persistent effects, the results do not strongly support a pure random walk specification. The estimated drift, on the other hand, stays almost identical in two alternative models.

\section{Conclusion}

In this study, we analyze the empirical implications of two commonly used specifications about the potential output growth rate. In a state space framework, neither of these specifications is fully supported by the empirical results. We also find that, for the U.S. economy, potential output growth rate deviates from its steady state level for substantially long periods.

\section{References}

Chui, C., Chen, G., 1991. Kalman Filtering with Real-Time Applications. Springer, Berlin.

Clark, P.K., 1987. The cyclical component of U.S. economic activity. Quarterly Journal of Economics 102 (4), $797-814$.

Gerlach, S., Smets, F., 1999. Output gaps and monetary policy in the EMU area. European Economic Review 43 (4-6), 801-812. Harvey, A.C., 1985. Trends and cycles in macroeconomic time series. Journal of Business and Economic Statistics 3, $216-227$. 
Kuttner, K.N., 1994. Estimating potential output as a latent variable. Journal of Business and Economic Statistics 12 (3), 361-368.

Orphanides, A., Van Norden, S., 2002. The unreliability of output gap estimates in real time. The Review of Economics and Statistics 84 (4), 569-583.

Watson, M.W., 1986. Univariate detrending methods with stochastic trends. Journal of Monetary Economics 18, 49-75. 